\title{
Enseigner la collaboration : retour d'expérience sur l'atelier de projet « architecture et empreinte sociétale »
}

\author{
JEAN-PHILIPPE POSSOZ \\ Uliège - Faculté d'Architecture - TEAM 11 ; 41, bd de la Constitution 4020 LIÈGE - Bât A5 \\ - Loc 0/09 jp.possoz@uliege.be
}

\section{Résumé}

L'architecture est une discipline du projet qui fait appel, dans des situations de conception complexe, à des compétences sociocognitives telles que l'écoute, le leadership, l'empathie, la médiation ... souvent peu explicitées dans les parcours de formation. Cet article propose de partager une expérience pédagogique visant à enseigner ces compétences par un dispositif pédagogique basé sur l'apprentissage expérientiel et le live project. En prenant appui sur cinq années de productions de rapports réflexifs par les étudiants, il tente de mettre en évidence les apprentissages effectifs et prises de conscience des étudiants sur les enjeux et compétences liés au travail collaboratif.

\begin{abstract}
Architecture is a discipline that uses, in complex design situations, socio-cognitive skills such as listening, leadership, empathy, mediation... These skills are often not very explicit in training courses. This article proposes to share a pedagogical experience aimed at teaching these skills through a pedagogical process based on experiential learning and live project. Based on five years of reflexive reports produced by the students, it attempts to highlight the actual learning and awareness of students on the issues and skills related to collaborative work.
\end{abstract}

\section{Mots-clés}

Architecture, enseignement du projet, collaboration, design \& build, live project

\section{Keywords}

Architecture, design teaching, collaboration, design \& build, live project 
Le présent article témoigne d'une démarche autoréflexive portant sur un dispositif pédagogique appelé « architecture et empreinte sociétale » destiné à des étudiants en architecture. Il interroge les effets d'une intention pédagogique qui vise, entre autres, à développer les compétences de l'étudiant en collaboration. En articulant dans un premier temps la notion de collaboration aux spécificités de la conception en Architecture et de son enseignement au travers des « ateliers de projet », le texte argumente de l'intérêt d'expliciter cette compétence aujourd'hui. Le dispositif pédagogique est ensuite contextualisé et décrit dans ses modalités afin de constituer un cas d'étude. Dans un second temps, la méthode faisant appel à des traces d'apprentissages est décrite et les résultats sont présentés et discutés.

\section{Problématique : la place de la collaboration dans les pratiques d'atelier de projet}

\subsection{Concevoir c'est collaborer}

La conception, activité centrale du projet, peut être vue comme un acte d'intelligence laborieux et collectif, contrairement à l'acte de création, plus individuel et spontané (Terrin, 2009). Cette vision d'un processus intrinsèquement collectif est partagée par Darses (2009) qui identifie, au sein d'un processus de conception, deux activités coopératives qui s'alternent: la coconception (synchrone) et la conception distribuée (asynchrone). Ainsi, concevoir implique des formes structurées d'interactions entre différents acteurs dont le niveau d'interdépendance (Little, 1990), s'il est intrisèquement fort, peut varier, selon les situations, entre coopération et collaboration.

Cette distinction entre travail collaboratif - au sens que lui ont donné de nombreux auteurs (Bélanger, 2017 ; Piquet, 2009) - et le travail coopératif est notable dans : les différences de relations existant entre les membres du groupe (liberté ou obligation) ; la responsabilité engagée de chacun par rapport aux actions (responsabilité partagée ou déléguée), et enfin par l'influence que chacun peut - ou ne peut pas - exercer sur la définition (quoi et pourquoi) et la succession des actions (comment) permettant d'atteindre l'objectif assigné au groupe. Le terme collaboration traduit ainsi «une forme d'organisation solidaire du travail où chacun est responsable pour le tout, sans que la part individuelle puisse être systématiquement isolée, la coordination se faisant par ajustement mutuel » (Gangloff-Ziegler, 2009). Il rend bien compte des interactions au sein d'un groupe de concepteurs dont l'homogénéité relative de l'expertise 
et des compétences initiales amène une plus grande difficulté dans l'attribution des tâches et une bataille dans la prérogative de «l'auteur », position jugée comme la plus élevée en terme de reconnaissance.

\subsection{Les enseignements du projet}

Les études d'architecture ont de tout temps accordé une grande place à « l'atelier». Ce mode d'enseignement immerge l'étudiant dans une situation professionnelle hypothétique et simplifiée, l'amenant à développer un projet. Cette simplification du cadre de questionnement diminue progressivement au fil du parcours d'étude mais maintient une forme de « caricaturisation » des contraintes propres à une situation professionnelle réelle.

Si les pratiques de Design \& Build, mêlant conception et réalisation, permettent un plus grand réalisme sur certains pans de contraintes (économiques, techniques, matérielles, logistiques...), ce sont les pratiques pédagogiques dites live project, plongeant l'étudiant dans une commande réelle, qui offrent le plus grand degré de contingences. Celles-ci font l'objet de nombreuses études, en particulier dans le monde anglo-saxon (Anderson, 2013, 2017 ; Brown, 2012 ; Rodriguez, 2018 ; Sara, 2004). Outre les interactions entre pairs, elles offrent à l'étudiant, selon le mode de cadrage choisi par l'enseignant, la possibilité d'une interaction avec des tiers (maître d'ouvrage, usager, administration...).

\subsection{Apprendre à collaborer : une hypothèse pédagogique}

\subsubsection{Pourquoi ?}

Durant les études d'architecture, où l'enseignement du projet est initié le plus souvent dès la première année, la collaboration (entre pairs), par le biais des travaux de groupes, est vue comme une activité évidente. Peu présente dans les référentiels comme un objectif à part entière, elle est souvent pratiquée de manière informelle et intuitive, se base sur des compétences implicites et ne fait que rarement l'objet de retours métacognitifs.

Cependant, comme toute activité collective, la collaboration renferme des enjeux sociorelationnels (leadership, identité, rapport de confiance, conflit interpersonnel...) qui impactent les interactions entre acteurs et les choix posés, tant individuellement que collectivement (Darses, 2009). La qualité de la production peut dès lors être fortement liée à la qualité du processus, il en va de même pour la qualité des apprentissages.

L'injonction à collaborer, présente dans de nombreux milieux aujourd'hui, n'est pas dénuée de valeurs (Lessard et al., 2009). L'enthousiasme autour des pratiques collaboratives traduit la 
nécessité de faire face à des problèmes de plus en plus complexes et urgents : les enjeux politiques, sociaux et environnementaux d'un côté (identité activiste), la compétitivité dans un monde en pleine tension de l'autre (identité managériale) (Sachs, 2001). Il semble ainsi que notre monde ait besoin d'individus capables de se mettre en lien, de penser et agir ensemble dans un dessein commun ; savoir collaborer est devenu une compétence reconnue et souhaitée. Apprendre à collaborer est donc devenu notre intention pédagogique centrale.

Avançons trois raisons de convoquer la collaboration dans nos pratiques d'enseignement du projet : premièrement celle de cultiver comme savoir-être notre capacité instinctive à la collaboration (Servigne et Chapelle, 2017); deuxièmement celle de développer, dans un temps donné, la capacité à prendre appui sur une lecture plus complexe du réel par la multiplication et le croisement des enquêtes et points de vue ; enfin celle de permettre l'exploration, au travers de la réalisation matérielle du projet au sein du temps pédagogique imparti, du lien entre pensée et action - faire soi-même, faire avec l'autre et faire faire par l'autre - comme des démarches indissociables s'alimentant mutuellement (Le Moigne, 2010).

\subsubsection{Comment?}

L'efficience de la collaboration au sein d'un groupe de travail dépend du nombre d'acteurs et du temps qu'ils peuvent consacrer à ce travail. Mais elle passe également par le respect et l'attention portés à certaines caractéristiques de base du groupe et de ses membres. Celles-ci, bien identifiées par Friend et Cook (2010) peuvent être résumées comme suit : le volontariat (nul ne peut être contraint à collaborer); la parité (égalité des parties, reconnue et maintenue tout au long du processus) ; le partage de buts mutuels, des responsabilités et des ressources ; la valorisation par chacun du modèle de collaboration; la confiance mutuelle et un sentiment d'appartenance au groupe.

Soutenir la collaboration invite ainsi à porter attention à la formalisation du cadre pédagogique, par exemple en garantissant : la libre participation (cours à choix), la non-hiérarchie (étudiants issus d'une même année d'étude), la limitation de la taille du groupe, l'adéquation entre le temps pédagogique et l'ampleur du projet pour que l'objectif soit ambitieux mais réaliste.

Il semble également nécessaire de soutenir le fonctionnement d'un groupe collaboratif durant le déroulement de l'activité. Servigne et Chapelle (2017) montrent en effet que les mécanismes de coopération bien que «naturels » et puissants, sont fragiles et nécessitent la mise en place d'équilibres, de règles et d'outils, afin de nous rendre compétents en coopération, de garantir 
un bon niveau de collaboration (ou un faible niveau d'égoïsme), et une bonne « écologie » du groupe et des individus qui le composent.

Sans qu'il y ait consensus sur la manière de procéder, un grand nombre d'auteurs reconnaissent ainsi la nécessité de mettre en place des dispositifs de soutien, tant matériels que méthodologiques (Galand et al., 2008). Ces dispositifs peuvent être convoqués selon leur capacité à faciliter un ou plusieurs des trois processus conjointement à l'œuvre au sein du travail collaboratif: la communication (modes d'échanges d'informations), la coordination (attribution et explicitation du rôle et des tâches de chacun et de tous) et la collaboration (modes d'interactions sur l'objet du travail) (Levan, 2020 ; Piquet, 2009).

Ces différents dispositifs de soutien facilitent l'émergence d'une forme de «conscience de groupe », notion qui comprend : la conscience de l'activité des autres, la conscience de la disponibilité des autres, la conscience du processus commun, la conscience des perspectives/buts et la conscience de l'environnement (Piquet, 2009).

\subsubsection{Question de recherche}

À l'intérieur du potentiel d'interactions que représentent les pratiques de projet, nous nous intéressons aux éléments du cadre pédagogique et aux dispositifs mis en place qui favorisent les apprentissages en matière de collaboration. Ainsi, la question de recherche qui en découle serait la suivante: comment une pratique pédagogique mise en place pour favoriser explicitement la collaboration entre pairs est perçue par les étudiants et comment cette perception informe la pratique pédagogique en retour? Ce texte propose d'explorer cette question au travers d'une pratique d'enseignement bien spécifique que nous allons maintenant nous attacher à décrire.

\section{Contexte : l'atelier de projet « architecture et empreintesociétale »}

\subsection{Cadrage et contextualisation}

L'atelier de projet « architecture et empreinte sociétale » est une unité d'enseignement à choix de huit crédits qui a vu le jour en 2014 à la faculté d'Architecture de l'Université de Liège et vivra en cette année 2021 sa dernière édition sous le format présenté ici. Alliant les notions de 
« conception orientée moyens » (Means Oriented Design) ${ }^{1}$, de « conception orientée usages » et de « conception collaborative », cet atelier tente au travers d'un processus de conception et de construction, d'ancrer la conception en architecture dans le réel. Il s'inscrit dans la catégorie des Live Projects impliquant un engagement en temps réel envers un client et des usagers tout aussi réels (Anderson, 2017 ; Brown, 2012; Sara, 2004) et rassemble chaque année une moyenne de 15 étudiants de Master 2 autour d'un projet sollicité par un commanditaire issu de la société civile ou d'un organisme public. Le cadre de l'activité est ainsi explicitement orienté vers une maximisation du potentiel d'interaction entre acteurs (voir la Figure 1).

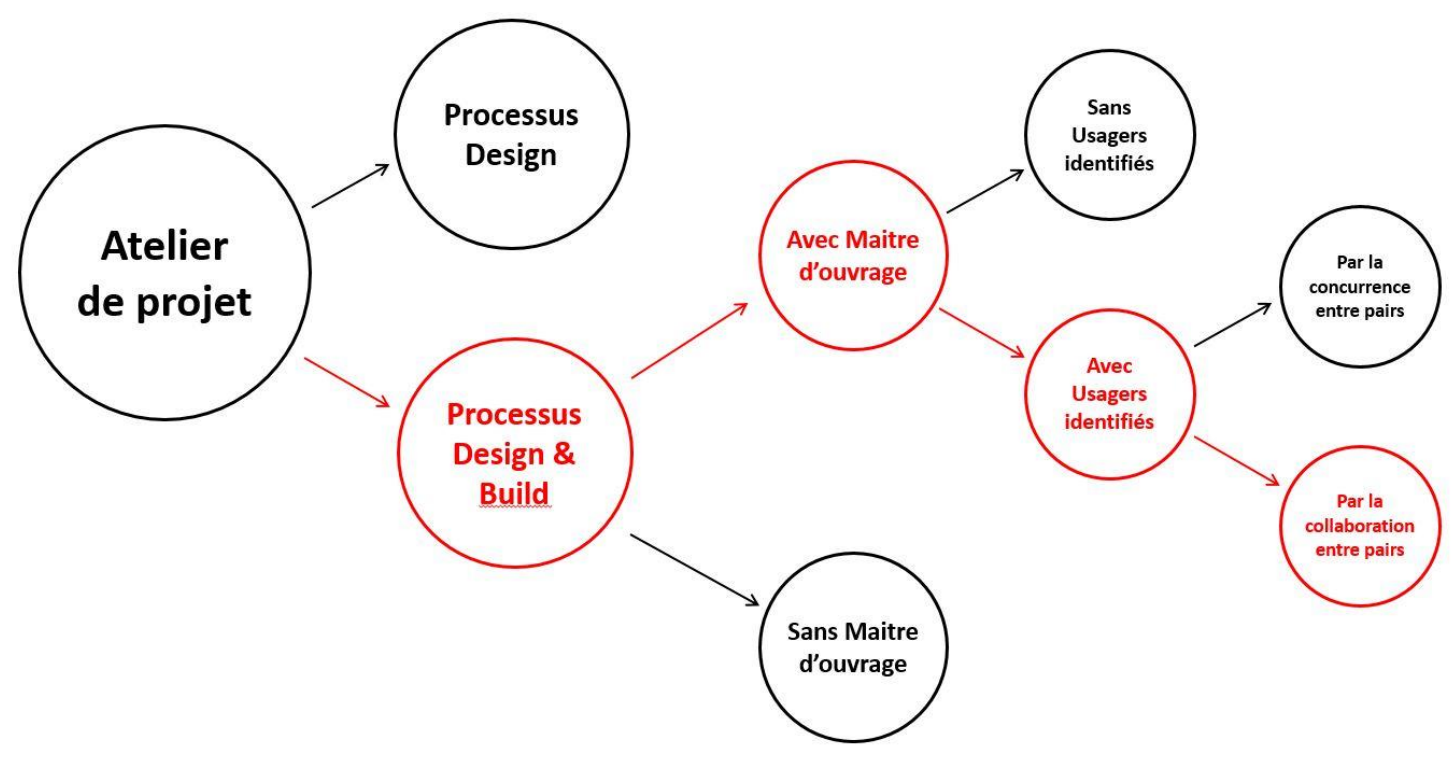

Figure 1 : Enchaînement des choix de cadrage impactant le potentiel d'exploration des interactions

Questionnant la responsabilité sociétale des actes en architecture, l'atelier se fonde sur l'économie de moyens comme prétexte à interroger tant le sens des réponses formulées au travers du projet, que les pratiques et les outils qui construisent ces formulations. Il invite chaque étudiant à se positionner comme acteur d'un processus et lui permet de percevoir comment influer, par sa propre posture, sur un processus multi-acteurs, en se mettant au service de l'efficience globale du résultat. L'objectif de réalisation soutient :

- la « conception orientée moyens » dans la mesure où la tension financière impose une parcimonie dans l'achat de matériaux et le choix de techniques ;

\footnotetext{
${ }^{1}$ Terminologie employée par TAEKE DE JONG dans l'ouvrage consacré au SUPERUSE STUDIO (Hinte et al., 2007).
} 
- la « conception orientée usages » car le résultat est évalué par les bénéficiaires du projet lors de l'inauguration ;

- la «conception collaborative » étant donné que l'accroissement du niveau de complexité pris en compte invite à une diversification des rôles et des tâches tout en maintenant l'objectif commun.

\subsection{Objectifs et Déroulement}

Au sein du référentiel de compétence de la formation, cet atelier met l'accent sur deux grandes compétences axées sur la mise en œuvre et les interactions comme des paramètres à part entière du projet. L'étudiant doit ainsi développer sa capacité à :

- identifier les enjeux, processus et acteurs d'une opération de construction et en déduire une posture adaptée ;

- utiliser de manière adéquate des outils aux services de pratiques collaboratives ;

- intégrer avec pertinence des enjeux environnementaux et sociétaux au processus de projet jusqu'à sa matérialisation ;

- choisir et élaborer des outils de médiation vers les constructeurs et utilisateurs en fonction des objectifs et enjeux spécifiques du projet (plan, croquis, cahier des charges, note, métré, norme, maquette, réunion, écolage, visite ...) ;

- $\quad$ se positionner face à la question du résultat (qualité, coût, durabilité, usage, plaisir, ...) et de sa maîtrise par l'architecte.

L'atelier fonctionne comme une agence dont le mode de gestion est basé sur la collaboration entre pairs. Il se déroule sur deux quadrimestres consécutifs durant lesquels le projet est nourri de semaines en semaines lors de séances collectives d'une durée de 4 heures, où s'alternent moments de synchronisation (émotionelle, informationelle, organisationelle) et travaux en petits groupes.

Librement inspirées des méthodes pédagogiques de construction du consensus développées par Badanes (2008), les méthodes proposées pour faire avancer le projet rejettent toute forme de mise en concurrence entre les membres du groupe, au profit de séances mobilisant des techniques de la sociocratie pour co-construire les décisions par l'adhésion de tous.

Des apports théoriques ainsi que des dispositifs de soutien aux pratiques collaboratives (voir l'Annexe 1) ont été introduits lors des trois dernières années dans le but d'améliorer l'efficience des moments de synchronisation cognitive et des moments de prise de décision :

- L'outil 1 : Les fondamentaux de la pratique de réunion collective, venant en soutien des processus de coordination et de collaboration ; 
- L'outil 2: Le tableau d'avancement du groupe, cherchant à faciliter le processus de communication ;

- L'outil 3 : Le point météo, permettant quant à lui essentiellement de soutenir les processus de communication et de collaboration.

Entre ces séances, des rôles et des tâches individuelles ou en sous-groupes sont collectivement identifiés, définis et attribués. Une consigne de roulement des attributions est également proposée afin de déjouer tout phénomène de «spécialisation ». Le déroulé théorique proposé est adapté chaque année avec les étudiants en fonction des spécificités du projet, de leur rythme d'avancement, de leur mode d'organisation. Il est formalisé comme suit :

1. Inscription: chaque étudiant transmet une lettre ouverte exprimant les raisons et questionnements qui le poussent à s'impliquer spécifiquement dans cet atelier ;

2. Mise en place du cadre ( 2 séances) : par des mises en situation, discussions et débats, aidé par des apports théoriques et des outils de soutien (voir l'Annexe 1), le groupe apprend à se connaître et à roder ses modes de fonctionnement. En parallèle, les enjeux de l'atelier sont explicités, débattus, redéfinis au regard des attentes du groupe ;

3. Conception (10 séances) : la lecture d'une lettre de commande et la rencontre avec le client marque le lancement de la conception qui se finalise par la présentation du projet aux futurs utilisateurs ;

4. Mise au point (4 séances) : le groupe travaille à la concrétisation du projet sur le plan technique, financier, matériel, logistique, culturel, etc. ;

5. Préparation du chantier ( 2 séances) : le groupe prépare l'intervention avec le client et les futurs intervenants ;

6. Réalisation ( 2 semaines de $40 \mathrm{~h}$ ) : chantier collectif et participatif où collaborent étudiants, techniciens et utilisateurs ;

7. Conclusion : l'atelier se conclut par un événement inaugural défini par le groupe en cours de projet.

\subsection{Dispositif d'évaluation}

Traditionnellement, l'évaluation des projets d'architecture se déroule lors d'un jury qui évalue le résultat du travail. Ici, le projet produit collectivement et fruit de l'implication individuelle de chaque étudiant est simplement montré et débattu lors de l'évènement inaugural.

Une double évaluation est proposée portant autant sur le résultat que sur le processus : l'une collective (auto-évaluation) sur base de critères prédéfinis (voir la Figure 2), l'autre individuelle sur base de l'observation par l'enseignant de l'implication et l'ajustement postural de l'étudiant d'une part et d'autre part sur base d'un rapport écrit, réflexif et critique, rédigé par celui-ci. 
1 Qualité du processus tant sur le plan organisationnel que relationnel

2 Capacité du résultat produit à rencontrer les attentes du client

3 Capacité du résultat produit à intégrer un propos environnemental et sociétal

4 Capacité du résultat produit à rencontrer le défi de l'économie de moyens

5 Capacité du groupe à restituer sa démarche au moyen d'un support média

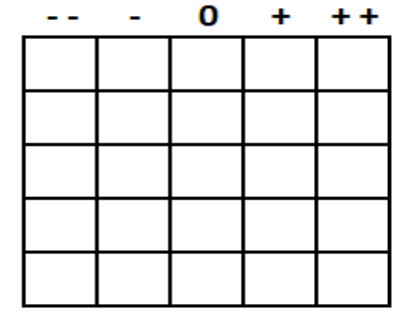

Figure 2 : Grille de critère d'auto-évaluation collective proposée par l'enseignant

Ce rapport intitulé « rapport individuel d'apprentissage » constitue la production ultime issue de l'atelier. Les consignes inscrites dans les engagements pédagogiques sont rappelées aux étudiants un mois après l'événement inaugural qui marque la fin du projet. Les consignes, assez simples, demandent à l'étudiant après relecture de sa lettre ouverte initiale, de produire un texte réflexif de l'ordre d'une page A4 recto-verso et développant :

- une position critique : sur les enjeux de l'atelier, le projet conçu et réalisé, la discipline de l'architecture, son action, ses enjeux, son enseignement, etc. ;

- une réflexion sur ses apprentissages personnels : de quoi est-il mieux (ou plus) capable (ou pas) ? Pourquoi selon lui, et en quoi cela lui semble plus ou moins important à ses yeux?

\section{Méthode}

Le point de départ de cet article réside dans la volonté de questionner notre propre pratique autant que d'en évaluer les effets. Cette démarche peut être qualifiée d'autoréflexive dans la mesure où ici, le chercheur, l'enseignant et l'encadrant ne font qu'un. Attentif à tous les biais propres à cette posture (Jorro, 2005), nous avons choisi de nous appuyer sur une partie des matériaux produits par les étudiants et qui attesteraient, dans une certaine mesure, de leurs apprentissages. Ainsi dans le cadre de cette publication, nous exploitons exclusivement les 76 rapports individuels d'apprentissage recueillis durant les cinq premières années d'encadrement de cet atelier (voir l'Annexe 2 pour un aperçu des 5 années concernées). Ils forment un corpus de données qui, même s'il doit être pris avec précaution (Bibauw, 2010), nous donne accès à une information réflexive sur les apprentissages. Ils témoignent de l'importance accordée par chaque étudiant à certains vécus émotionnels, certains constats, liés à leur apprentissage durant l'année mais également pour certains, un recul critique sur la formation reçue durant cinq années.

L'exploration des rapports d'apprentissage comme source d'une démarche autoréflexive sur plusieurs années de pratique d'enseignement est une intention qui naît a posteriori. En ce sens le matériau (les rapports) n'a pas été «formalisé » au départ pour obtenir des données. Nous 
pourrions ainsi formuler notre démarche comme une tentative de réflexion sur «l'action d'enseigner » se basant sur les traces et indices d'apprentissages décelés au sein d'un corpus de réflexion sur « l'action d'apprendre ».

De ce matériau, nous traitons ici exclusivement la question de la « conception collaborative » et des apprentissages spécifiquement liés à la collaboration entre pairs au sein de l'activité de conception. Bien que de nombreuses traces témoignent d'apprentissages relatifs à la « conception orientée moyens » ou à la «conception orientée usagers », celles-ci ont été volontairement laissées de côté afin de garder la focale sur la collaboration entre pairs.

Notre approche est essentiellement qualitative. En effet, bien que les traitements des données aient fait usage de recherches d'occurrences par mots clefs qui ont d'ailleurs influencé le mode d'interprétation, il est apparu que cette méthode laissait de côté un grand nombre de constats signifiants. Ainsi, prenant appui sur la notion de significant learning de Fink (2003), la sélection des extraits prélevés du matériau de base a mis l'accent sur les traces de sens accordé par l'étudiant, au travers des mots, à des moments et des dispositifs, tout en étant attentive à la perception de changement chez l'étudiant.

\section{Résultats et interprétations}

La phrase suivante illustre le conflit cognitif auquel les étudiants font face en début d'année :

"Mais comment concevoir de tels projets à 15 personnes alors que nous sommes habitués à créer individuellement, voire en très petits groupes? "².

La situation pédagogique interpelle, elle rend inopérante la reproduction du mode de pratique antérieur et semble inviter l'étudiant à investir le processus collaboratif comme enjeu à part entière. Nous allons dans un premier temps déplier, en articulant citations et interprétations, les nombreuses traces de cet investissement qui relèvent de la collaboration entre pairs avant de revenir, selon le même procédé, sur les quelques éléments qui informent sur la place de l'enseignant dans ce processus et la perception des étudiants relative au mode d'intervention tutorale.

\subsection{La collaboration entre pairs}

\subsubsection{Construction d'une identité commune}

«C'est une des choses le plus à retenir de l'atelier : le Groupe ».

\footnotetext{
${ }^{2}$ Les phrases entre guillemets et en italique sont toutes extraites des rapports d'apprentissage.
} 
La notion même de groupe est régulièrement mise en évidence, il devient un individu à part entière, il possède une entité à laquelle chacun finit par se référer. L'importance accordée à sa formation, son émergence au travers des rapports informels, est mise en avant :

"Un travail de groupe qui fonctionne dépend aussi certainement d'une cohésion. Des moments de relâchement ensemble n'ont fait qu'accentuer l'idée de "groupe". ".

Une conscience de la nécessité de développer des " outils propres à la cohésion de groupe » apparaît également bien que moins régulièrement. Cela peut s'expliquer en partie par le fait que l'outillage des pratiques est apparu progressivement (voir l'Annexe 1), son introduction par l'enseignant étant, dans les faits, une forme de régulation.

Le fait de travailler pour les autres autant que pour soi participe à l'émergence d'une motivation intrinsèque orientée autour des objectifs fixés par le groupe :

«Nous avons tous eu la sensation de travailler dans une équipe plus que pour un cours. ";

"Quelle satisfaction ressentie lors de l'inauguration quand j'ai regardé le projet fini et me suis dit on l'a fait, on y est arrivé, tous ensemble et grâce à tout le monde. ».

Cette identité collective ne semble pas induire de réduction du sentiment de responsabilité individuelle sur le résultat produit. Une étudiante exprime bien cette translation $\mathrm{du}$ « rapport émotionnel au projet » passant de l'individuel au collectif :

"J'ai surtout appris à prendre du recul quant au projet que je, ou plutôt que l'on développait. Faire en sorte de lâcher "mon bébé”, et de ne plus m'attribuer un projet comme étant le mien, mais comme étant le nôtre. ».

Cette identité commune est également associée à une réalité professionnelle qui nécessite la mobilisation de compétences spécifiques, la collaboration étant identifiée comme :

« une logique de travail plus proche de celle propre à notre métier c'est-àdire celle d'un groupe, d'un bureau d'architecture où chacun collabore et amène des idées. ".

\subsubsection{Intersubjectivité}

En situation de conception collaborative, l'égo individuel est autant un atout qu'un frein. La place des personnalités dans les groupes questionne les étudiants durant l'élaboration du projet. Soutenus par une certaine empathie pour le groupe, ils opèrent des ajustements posturaux plus ou moins conscients. Une négociation intérieure semble s'opérer : 
" J'avoue, qu'à certains moments, ce fut compliqué pour moi de mettre mon énorme égo de côté [...] si nous n'avions pas travaillé tous ensemble, nous ne serions jamais arrivés à un si beau résultat. " ;

"Me tempérer et laisser d'autres diriger et me guider, m'a permis d'en apprendre beaucoup sur la richesse qui se cache en chacun lorsqu'il se sent investi. ».

Les personnalités les plus fortes affirment opérer un travail sur eux-mêmes pour « gagner en tolérance » :

" Je pensais que ceux qui n'osaient pas parler n'avaient rien à dire. Une fois notre groupe soudé, ces personnes ont pu proposer leurs idées en toute confiance pour faire avancer nos réflexions. C'est probablement l'apprentissage qui me plaît le plus, le savoir-écouter. ».

Les personnalités plus effacées expriment également ces ajustements :

«En étant une personne très calme et timide, le simple fait de prendre la parole devant tout le monde représente pour moi un grand défi à surmonter. Grâce au climat du groupe et à l'application des règles de la pratique collaborative, j'ai franchi ce blocage par moment. ».

La conscience de « soi au sein du groupe » et de la finalité du projet les conduit à :

"veiller sur le bien-être des autres pour permettre des rapports autres que l'efficacité. " ;

" mieux écouter, prendre en compte les remarques de chacun. Partir d'une idée, (sur laquelle je n'étais pas convaincue), la retravailler, la faire évoluer jusqu'à un résultat qui était plus correct à mes yeux. » ;

" discuter sans chercher à être au-dessus du lot mais plutôt en cherchant à ouvrir un débat constructif et bénéfique à un projet commun à tous. ».

La dimension humaine des apprentissages au sens de Fink (2003), ici fortement illustrée, se révèle progressivement, soutenue par des moments de synchronisation émotionelle (Outil 3) qui participent à la construction de la consience de l'autre autant que du rapport de confiance :

"Le point météo a permis à chacun d'exprimer son ressenti et de mettre à plat des remarques qui n'auraient peut-être jamais été dites en dehors. ».

\subsubsection{Outils et méthodes}

L'investissement dans la construction de la collaboration - en tant que tâche à part entière - est un des éléments les plus laborieux à obtenir en cours de processus, tant l'envie que le projet avance mobilise l'attention. Cependant, les étudiants identifient a posteriori l'importance du processus de coordination : «La bonne coordination de chacun est un travail en soi ... », et son intérêt : 
«Construire sa propre hiérarchie de groupe et sa méthodologie d'action permet un investissement de chacun. ».

Bien que l'outil tableau de bord (Outil 2) ne soit jamais cité, les étudiants se montrent conscients de la dimension centrale du processus de communication, du partage de l'information et de son outillage, en ce compris lors des phases de travail asynchone pour lesquelles ils développent leur propre outil :

"pour la communication “extra-atelier”, l'outil de communication via facebook a pour moi très bien fonctionné. ».

Si les outils proposés par l'enseignant ne sont pas souvent mentionnés dans les rapports, les étudiants expriment néanmoins une conscience que le travail de construction du groupe reste fragile et mérite d'être investi :

"Travailler en groupe demande une gestion de groupe très organisée, des théories de dynamique de travail en groupe, une remise en commun permanente des idées, une compréhension des autres et un effort de communication, tous ces points, ont manifestement et durablement enrichi mon savoir personnel. ».

\subsubsection{Prise de décision}

Les moments de synchronisation, proposés chaque semaine en début et en fin de séance, servent autant à homogénéiser le niveau de connaissance et de conscience de l'avancement du travail qu'à travailler ensemble à l'élaboration du projet. Ces moments, où l'on fait avancer le projet ensemble, cristallisent toutes les tensions. Les consignes, visant à mobiliser les techniques de la sociocratie pour construire le consensus (Outil 1), semblent progressivement porter leurs fruits :

"La nécessaire prise de décision me paraissait parfois laborieuse, mais les tables rondes, les concertations, les réunions ont permis d'effacer cette idée qui a fait place à un processus, à une rythmique bien huilée aboutissant à des choix représentant un groupe soudé. ».

Cette pratique se heurte au départ à une très faible culture des étudiants au débat contradictoire vertueux. Signe que l'intérêt d'un rapport dialectique de qualité, proche de l'intelligence collective est peu ancré dans leur bagage d'apprentissage :

«CONSTRUIRE une pensée en groupe... la pensée et le travail en groupe sont trop souvent assimilés dans nos études, à des confrontations d'idées, jugées et étiquetées comme bonnes ou mauvaises par nos pairs. Ici, et bien que ce fut une des choses les plus difficiles à obtenir, les idées sont écoutées et réfléchies de manière à prendre ce qu'il y a de bon et de mauvais pour en 
arriver à un consensus de pensée collective. L'ouvrage final et la réflexion globale proviennent d'un accord commun. ».

Cette difficulté à faire avancer le projet est présente dans de nombreuses traces mais, loin de rebuter, elle semble fondatrice à certains :
"S'il faut retenir quelque chose selon moi, c'est le fait de concevoir un projet, par un seul et même groupe, évitant ainsi toute concurrence entre les étudiants. ».

Lors d'un atelier (en fin de phase de conception), l'enseignant propose au groupe de mettre en place des critères d'évaluation communs. Cette co-construction des critères de jugement aidant à la prise de décision :

«Lors des échanges de groupe, quand il fallait faire des choix par rapport à des critères communs et non pas avec des inspirations personnelles. ».

\subsubsection{Répartition des tâches et des rôles}

Une consigne de roulement des tâches et des rôles par adhésion et « don de soi » et non par compétence ou affinité est proposée au groupe. Cette consigne, au départ abordée avec réticence, est débattue par les étudiants et l'intérêt de cette déstabilisation est progressivement identifié :
"On a tous compris que c'était lors des changements de groupe que les idées venaient plus naturellement. Quand on travaillait en groupe figé, on perdait du temps et les idées étaient beaucoup moins riches. " ;
"Il a parfois été difficile d'accepter que nos idées soient critiquées et même complètement changées lorsqu'un autre groupe reprenait un projet en cours. ».

Des limites à cette rotation des rôles sont soulevées :

«Cela a peut-être aussi posé problème parfois, dans le sens où il n'y avait pas de personne référente. ".

Mais par contraste, son intérêt fondamental est néanmoins reconnu :

« Nous aurions pu tomber dans le cliché de séparer les tâches par catégories et que chacune d'entre elles soit dirigée par une personne (chef). Cette organisation aurait généré en nous un sentiment d'appropriation très fort et une compétitivité mal venue. Le principe itératif dans lequel nous nous sommes engagés était plus intéressant car il nous permettait de toucher à une plus grande partie de la conception. ».

Le passage à l'acte de construire cristallise en général l'énergie du groupe, très désireux de voir un projet concrétisé. Cette soif de faire amène à une chute dans la collaboration. Elle découle 
de deux facteurs, l'envie de maîtriser le résultat et l'envie d'explorer certaines techniques ou certains matériaux :

"Sur le chantier, avec la pression, certains d'entre nous se sont plus attelés à une tâche selon leur préférence ou selon leur aptitude manuelle dans le domaine où ils se sentaient le plus à l'aise. ».

La rotation des tâches impose de porter une attention sur l'identification des intentions et leurs transmissions, elle entraîne aussi un effet collatéral, le détachement du lien individuel au résultat produit, au bénéfice du lien collectif :

"On continue le travail de quelqu'un, et on accepte et on laisse quelqu'un reprendre le notre, et se l'attribuer. Au final du chantier, le travail reconnu était le travail d'un groupe et pas d'une seule personne. ».

\subsection{Le rôle de l'enseignant}

Accompagner ce type de processus pédagogique nécessite de la part de l'enseignant une négociation posturale permanente, à la fois en dedans et en dehors. Le rôle assigné explicitement dans l'engagement pédagogique (avocat du maître d'ouvrage et expert technique) est bien en deçà des besoins en soutien identifiés en pratique et exprimés par les étudiants.

Ils qualifient le rôle de l'enseignant par des adjectifs tels que : "enrôleur, initiateur, manager, médiateur, stimulateur et contrôleur, sans jamais être dictateur. " Laissant apparaître la diversité des modalités d'interventions tutorales, réactives et proactives, qu'ils lui attribuent.

Lors des prises de décision, la position de retrait par rapport aux choix opérés par le groupe est lue comme une forme d'autonomie négociée, une confiance accordée au groupe :

«Nous étions libres de nos choix puisque vous vous placiez en retrait le plus souvent possible afin de nous rendre responsables de nos décisions. ».

Ils identifient la fragilité inhérente au modèle pédagogique en le qualifiant d' efficace et risqué, mais qui a porté ses fruits », ainsi que les tenants et aboutissants d'une forme de droit à l'erreur :

"Faire des erreurs pour en tirer nos propres conclusions." "Nous avons avancé par essais-erreurs mais ce qui est important est que nous nous sentions malgré tout en sécurité. ".

Ils identifient les moments où une intervention est opérée pour " remettre les pieds sur terre », ou « avancer ensemble et pas les uns contre les autres. ». 
Ils expriment la dimension désirable d'une forme d'enseignement favorisant l'autonomie et la responsabilisation :

\begin{abstract}
"Nous sommes progressivement amenés à organiser les choses par nousmêmes sans avoir continuellement un rapport prof-élève mais plutôt la formation d'un groupe de travail avec une personne de référence qui nous guide lorsque c'est nécessaire [...] cette forme d'enseignement doit être encouragée, de manière à plus responsabiliser les étudiants. ».
\end{abstract}

\title{
5. Discussion
}

La diversité des enjeux de l'exercice et les consignes lâches fournies pour l'écriture du rapport d'apprentissage n'amenaient pas l'étudiant à identifier la collaboration entre pairs comme un item incontournable lors de la rédaction du rapport. Sans prétendre éviter tout biais de désirabilité, cette disposition semble avoir permis, dans une certaine mesure, de limiter le problème du formatage des réponses tel qu'évoqué par Bibauw (2010).

Le nombre et la diversité des traces relevées qui commentent ce sujet nous semblent donc en mesure d'attester, à tout le moins, d'une prise de conscience largement acquise des enjeux du processus de collaboration entre pairs. Au-delà, un changement notable apparaît chez les étudiants dans leurs rapports au projet et à l'activité de conception elle-même. L'insertion explicite de la collaboration entre pairs, parmi les enjeux de l'activité, modifie chez certains étudiants au moins la perception de l'objectif à atteindre. La qualité du processus, et singulièrement la qualité des interactions au sein de celui-ci, semblent devenir importantes à leurs yeux. La relation à l'autre s'avère prendre une place importante dans les apprentissages, la notion d'ajustement postural nous paraît la plus à même de décrire ce qui s'opère chez l'étudiant qui renonce à remporter la bataille pour inviter l'autre à influer sur le projet.

Parmi les éléments du cadre qui ressortent, du point de vue des étudiants, comme favorisant la collaboration tout au long du processus, on retrouve l'attention apportée à la construction d'un bon « climat socio-relationnel» (Quintin et Masperi, 2010) au sein du groupe. Celle-ci passe par des dispositifs informels - ces «moments de relâchement» (pauses ludiques, rencontres hors cadre...) - autant que formels comme le point météo, qui concourent à consolider les liens interpersonnels autant que la « conscience de groupe ».

Y participe également un certain nombre de dispositifs de soutien, initiés par l'enseignant, et dans lesquels il apparaît a posteriori éclairant de distinguer : des outils formels, des consignes opérationelles et des modalités d'interventions tutorales (manières de mobiliser outils et 
consignes autant que de partager une expertise). Parmi ceux-ci, le rejet de toute forme de mise en concurrence et la formalisation des moments de prise de décision par des consignes évitant explicitement la formation de minorité (le vote) sont perçus comme favorables à la collaboration et à la motivation. La déconstruction, forcée par l'enseignant, d'une logique de répartition des tâches proche des mécanismes de coopération, par un roulement des rôles et tâches tout au long du processus, est ressentie comme favorable au maintien d'un niveau élevé de collaboration tout en soutenant l'acquisition par tous d'autres types d'apprentissages (capacité à déléguer, transmettre une information, prendre en compte le point de vue de l'autre...).

Peut-on pour autant conclure, à travers ces traces d'apprentissages, que ces étudiants soient tous devenus compétents en collaboration ? Il semble ici délicat de répondre par l'affirmative. Si la dimension expérientielle de l'exercice a indéniablement ancré des bribes de savoir-être et de savoirs procéduraux, leurs ancrages et leurs généralisations à tous les participants restent totalement incertains. L'analyse des traces d'apprentissages nous permet malgré tout de faire l'hypothèse que les éléments évoqués ci-dessus constituent autant de composantes d'une telle compétence.

Il ressort aussi de l'analyse que le modèle de tutorat choisi et les modalités d'interventions tutorales opérées durant le processus sont perçues par les étudiants comme participant à la construction de leur motivation et donc au maintien du sens. Si cette information ne relève pas strictement de la relation entre pairs, les témoignages démontrent qu'elles affectent également le climat socio-relationnel au sein du groupe d'étudiants. Ce qui peut laisser penser que l'enseignant, selon le mode de régulation qu'il emploie, est à certains moments perçu comme faisant partie du groupe.

Enfin, les rapports d'apprentissages sur lesquels nous nous sommes basés sont une forme de « réflexion sur l'action », elle vient a posteriori. Le plus faible nombre de retour sur les outils de soutien aux pratiques collaboratives tels que la co-construction de critères d'évaluation, l'outillage des moments de synchronisation ou le tableau visant à la synchronisation des informations, nous laisse penser qu'une forme plus régulière de « réflexion dans l'action » (Perrenoud, 2004) pourrait favoriser une meilleure appropriation des outils et méthodes proposés. 


\section{Conclusion}

Si nous pouvons affirmer qu'en apprenant en collaborant il est possible d'apprendre à collaborer, soutenir cet apprentissage invite tout au moins à le rendre explicite pour les étudiants. L'analyse du dispositif pédagogique présenté ici nous conduit à aller plus loin et émettre l'hypothèse que la qualité de ces apprentissages est conditionnée par au moins deux vecteurs. D'une part, un portefeuille d'outils et méthodes, ajusté et négocié selon les situations, qui constitue un cadre favorable et qui mérite d'être régulièrement remobilisé lors de moments réflexifs au cours du processus. D'autre part, une position négociée de l'enseignant, sensible au climat socio-relationnel du groupe, à l'écoute de la manière dont les étudiants s'approprient ces outils et méthodes. Ainsi, pour reprendre le mot d'un étudiant, c'est une pratique "risquée » mais dont le potentiel émancipateur transparaît au travers des traces. En ce sens, si elle peut s'avérer inspirante, elle ne peut se constituer en modèle.

Dans l'enseignement de l'architecture, le live project constitue indéniablement un bon dispositif pour le développement de compétences en collaboration, il cristallise les valeurs communes du groupe, soutient une motivation et un sentiment de responsabilité collective dont le point d'orgue est la réalisation du projet. Sans chantier, sans inauguration finale, les retours sur la collaboration ne seraient probablement pas les mêmes. Ce constat invite l'enseignant à porter une attention toute particulière à l'adéquation entre «temps pédagogique » et «temps du projet ». Il invite également à négocier avec le droit à l'erreur qui, s'il constitue un levier d'apprentissage puissant en cours de processus, peut se révéler contre-productif et démobilisateur si le résultat produit n'est pas à la hauteur des ambitions :

$$
\text { « Comme toute bonne expérience, au final on en retient que le meilleur! ». }
$$

À la lecture des rapports des étudiants, on peut supposer que ces expériences, qui les ouvrent aux enjeux qui se cachent au sein du processus de conception, laisseront des traces dans leurs futurs pratiques et choix professionnels.

\section{Références bibliographiques}

Anderson, J. (2013). Undercurrent: Swimming away from the design studio. Proceedings of the first international conference of the Association of Architectural EducatorsAAE:(un) common currency. Nottingham Trent University, UK.

Anderson, J. (2017). Devising an inclusive and flexible taxonomy of international live projects. ARENA Journal of Architectural Research, 2(1), 3. 
Badanes, S. (2008). Building consensus in design/build studios. Dans B. Bell et K. Wakeford (dir.). Expanding Architecture: Design as activism (p. 248-255). New York: Metropolis Books.

Bélanger, G. (2017). Qualité des pratiques de développement des compétences informationnelles au sein du réseau de l'Université du Québec|PDCI.

Bibauw, S. (2010). Écriture réflexive et réflexion critique dans l'exercice du compte rendu. Revue internationale de pédagogie de l'enseignement supérieur, 26.

Brown, J. B. (2012). A critique of the live project. Thèse de doctorat. Queen's University Belfast.

Darses, F. (2009). Résolution collective des problèmes de conception. Le travail humain, $72(1), 43$.

Fink, L. D. (2003). Creatin significant learning experiences. An integrated approach to designing college courses. New-Jersey: Jossey-Bass.

Friend, M. et Cook, L. (2010). Interactions: Collaboration skills for school professionals. White Plains, NY: Longman.

Galand, B., Bourgeois, É., Frenay, M. et Bentein, K. (2008). Apprentissage par problème et apprentissage coopératif : vers une intégration fructueuse ? Dans Y. Rouiller et $\mathrm{K}$. Lehraus (dir.), Vers des apprentissages en coopération : rencontres et perspectives, (p. 139-163). Berne : Peter Lang.

Gangloff-Ziegler, C. (2009). Les freins au travail collaboratif. Marche et organisations, 10(3), 95-112.

Hinte, E. van, Peeren, C. et Jongert, J. (2007). Superuse: Constructing New Architecture by Shortcutting Material Flows. 010 Publishers.

Jorro, A. (2005). Réflexivité et auto-évaluation dans les pratiques enseignantes. Mesure et Évaluation en Éducation, 27, 33-47.

Le Moigne, J.-L. (2010). Agir $\Leftrightarrow$ penser en complexité, le discours de la méthode de notre temps. Conférence Grand débat.

Lessard, C., Kamanzi, P. C. et Larochelle, M. (2009). De quelques facteurs facilitant l'intensification de la collaboration au travail parmi les enseignants : Le cas des enseignants canadiens. Éducation et Sociétés, 23, 59-77.

Levan, S. K. (s. d.). Ce que travail collaboratif veut dire... Le Travail Collaboratif en Ligne selon MAIN CONSULTANTS. Consulté 10 décembre 2020, à l'adresse https://ravailcollaboratif.typepad.com/methode_main/2009/03/ce-que-travailcollaboratif-veut-dire.html

Little, J. W. (1990). The persistence of privacy: Autonomy and initiative in teachers' professional relations. Teachers College Record, 91(4), 509-536.

Perrenoud, P. (2004). Adosser la pratique réflexive aux sciences sociales, condition de la professionnalisation. Éducation permanente, 160(3), 35-60.

Piquet, A. (2009). Guide pratique du travail collaboratif: Théories, méthodes, et outils au service de la collaboration. Département LUSSI.

Quintin, J.-J. et Masperi, M. (2010). Reliance, liance et alliance : Opérationnalité des concepts dans l'analyse du climat socio-relationnel de groupes restreints d'apprentissage en 
ligne. Alsic. Apprentissage des Langues et Systèmes d'Information et de Communication, 13.

Rodriguez, C. M. (2018). A method for experiential learning and significant learning in architectural education via live projects. Arts and Humanities in Higher Education, $17(3), 279-304$.

Sach, J. (2001) Teacher professional identity: Competing discourses, competing outcomes. Journal of Educational Policy, 16(2), 149-161.

Sara, R. (2004). Between studio and street: The role of the live project in architectural education. Thèse de doctorat. University of Sheffield.

Servigne, P. et Chapelle, G. (2017). L'entraide : L'autre loi de la jungle. Paris : Les liens qui libèrent.

Terrin, J.-J. (2009). Conception collaborative pour innover en architecture. Paris : L'Harmattan. 


\section{ANNEXE 1 : Portefeuille d'outils proposés au groupe}

Suite aux deux premières éditions de l'atelier, il est apparu souhaitable de soutenir plus explicitement le travail lors des moments de synchronisation et d'échanges en groupe où sont discutées des questions qui regardent autant l'organisation du travail que l'avancement et l'orientation du projet. Un document sur les enjeux des pratiques collaboratives a ainsi été rédigé à destination des étudiants. Celui-ci est partagé et débattu en séance et propose au groupe deux premiers outils.

\section{OUTIL 1 : Les fondamentaux de la pratique de réunion collective}

Lors des réunions de synchronisation, il est essentiel de respecter les consignes suivantes :

1. Définir l'objectif de la réunion : Pour économiser temps et énergie, il est important de se poser deux questions : Pourquoi se réunit-on? qui doit participer?

2. Définir le temps qui va y être consacré : Rien n'est plus énervant que des réunions qui n'en finissent pas.

3. Se disposer en cercle : On ne fait pas de bonne réunion entre deux portes, le dispositif spatial doit faciliter la participation et l'attention de tous.

4. Pratiquer le tour de parole : Lorsque certains s'accaparent la parole, la motivation et l'attention des autres disparaît progressivement.

5. Solliciter la parole plutôt que de l'imposer et faire en sorte que les opinions s'additionnent au lieu de s'opposer.

6. Placer la parole au centre : prendre l'habitude de s'adresser au groupe et non à une personne en particulier.

7. Nommer un gardien du cercle et des règles qui modère la parole et éventuellement assure l'avancement de la réunion.

8. Préférer obtenir des décisions par adhésion et consentement plutôt que par vote (Sociocratie versus démocratie)

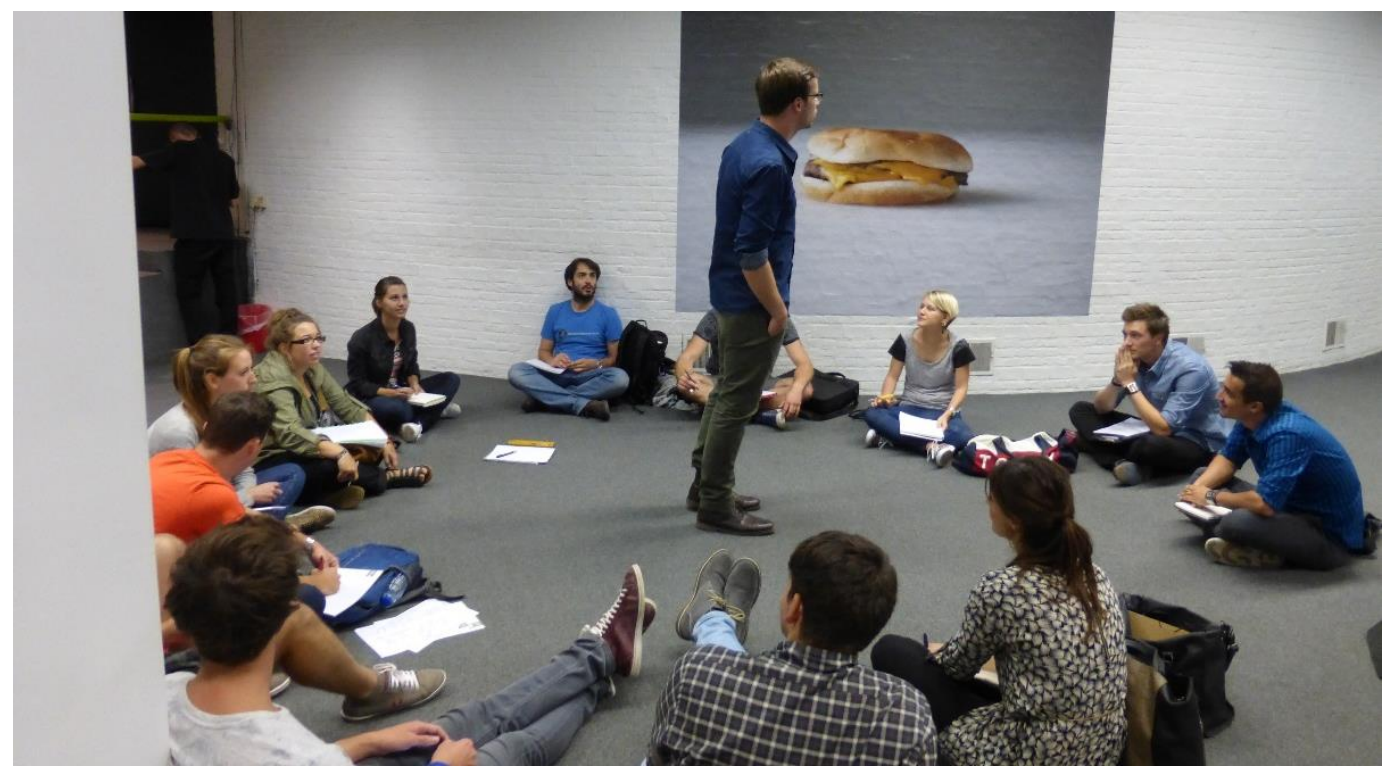

Réunion autour de la problématique de l'approche du contexte de projet 


\section{OUTIL 2 : Le tableau d'avancement du groupe}

Lors de chaque moment de synchronisation, il en va de la responsabilité de chacun et de tous de fournir à l'ensemble du groupe les éléments permettant de s'approprier collectivement l'avancement du travail. Un tableau d'avancement peut être mis en place afin d'organiser les éléments produits par chacun. Ces documents peuvent avoir plusieurs niveaux de lecture (synthèse graphique ou écrite, texte de référence, image, matériau, maquette, vidéo...) permettant ainsi à chacun de capturer rapidement le sens tout en pouvant approfondir au besoin.

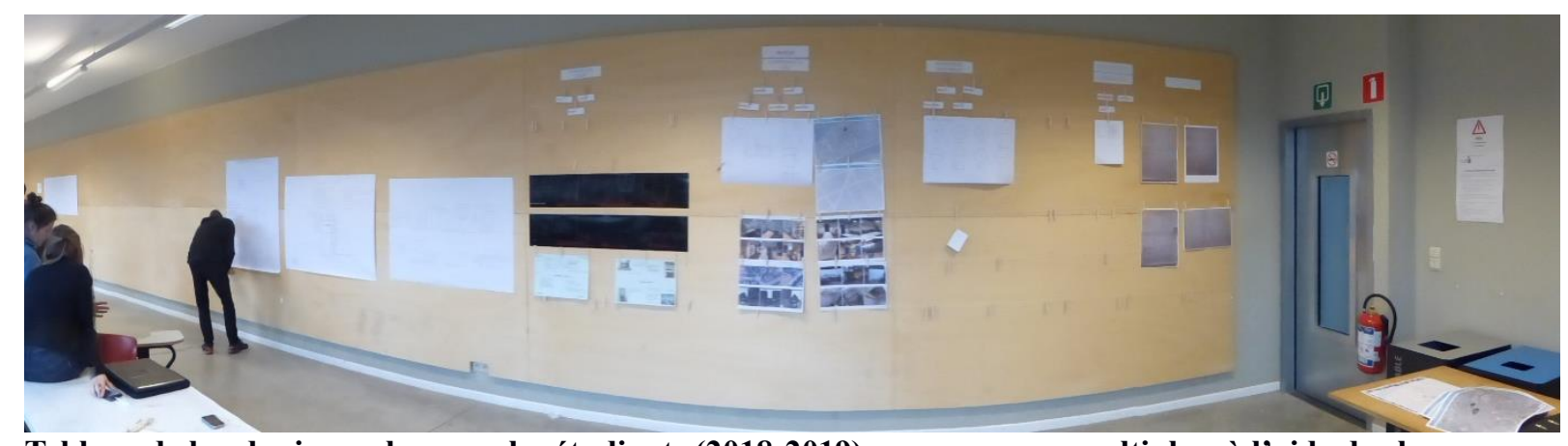

Tableau de bord mis en place par les étudiants (2018-2019) sur panneaux multiplex, à l'aide de clous, cordes de chanvre et pince à linges.

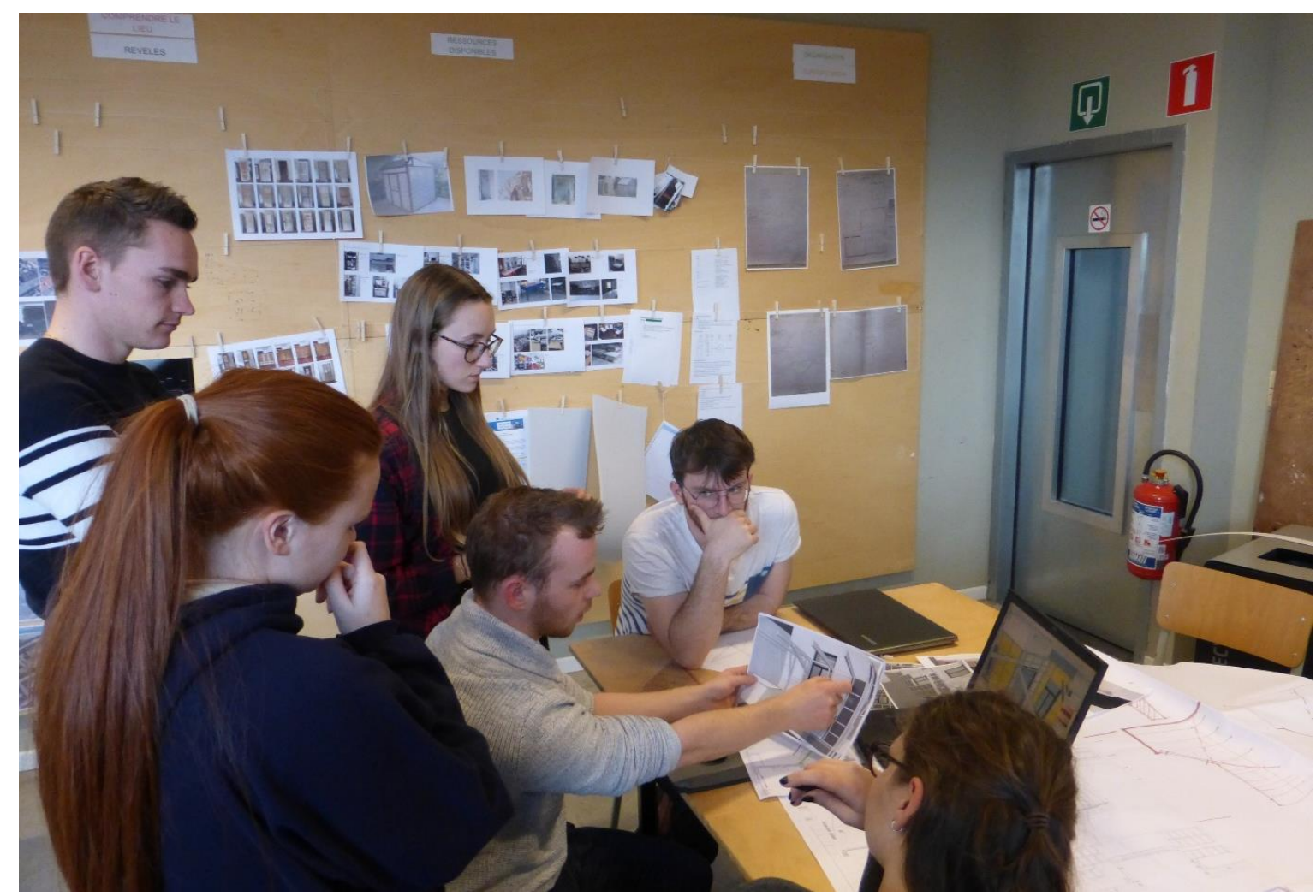

Travail en sous-groupe avec prise d'appui sur des documents issus du tableau de bord.

\section{OUTIL 3 : Le point météo}

Lors des moments de travail collectif, il est parfois important de partager l'état émotionnel des membres du groupe afin de favoriser une bonne qualité des échanges et du travail de collaboration. Pour ce faire, un tour de table sous forme de métaphore météorologique peut être fait afin de permettre à chacun de partager son état émotionnel (fatigue, enthousiasme, lassitude, excitation, énervement...) autant que d'entendre celui des autres. 


\section{ANNEXE 2 : Listes des terrains et illustrations}

2014-2015 - Centre culturel des Chiroux : accueil et espace foyer
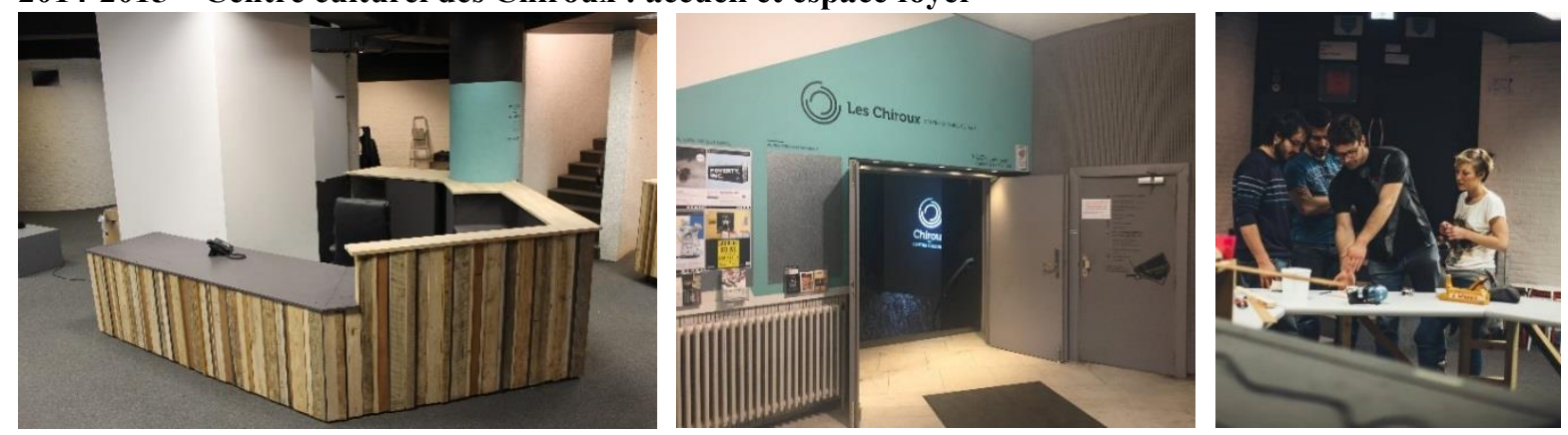

2015-2016 - Coopérative immobilière Dynamocoop : espace mutualisé Dony
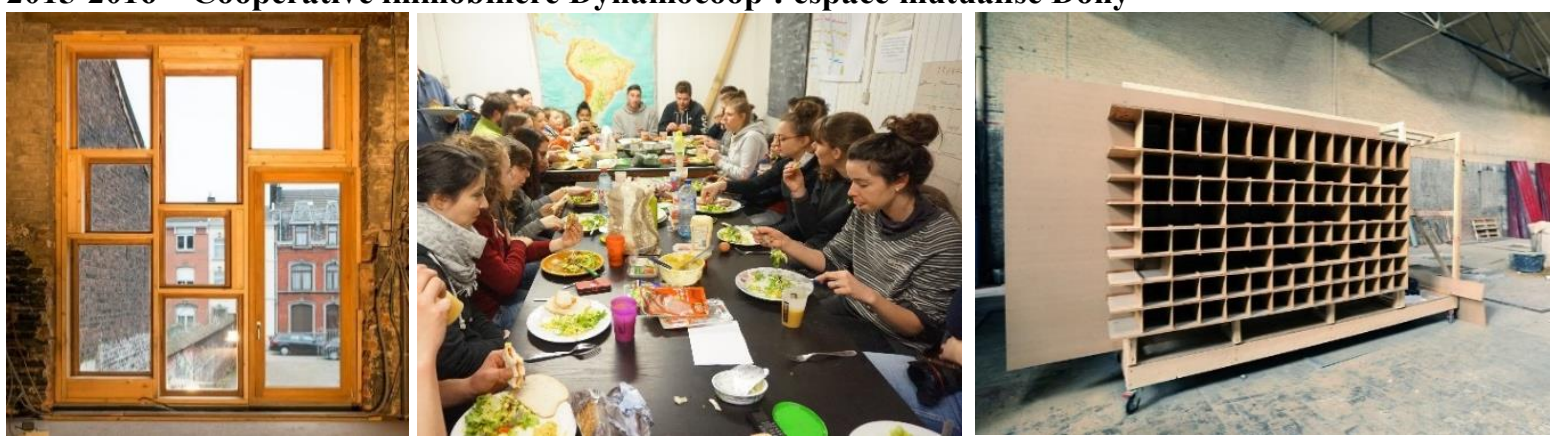

2016-2017 - Ville de Liège : Potager collectif des forges
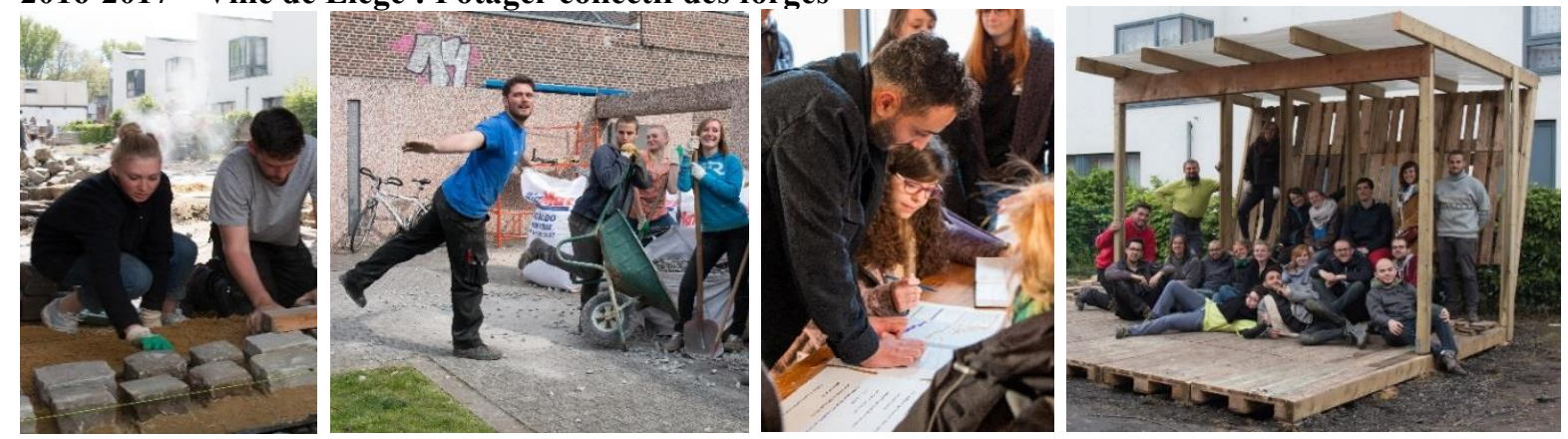

2017-2018 - Habitat-Service asbl : cour collective et espaces de travail social
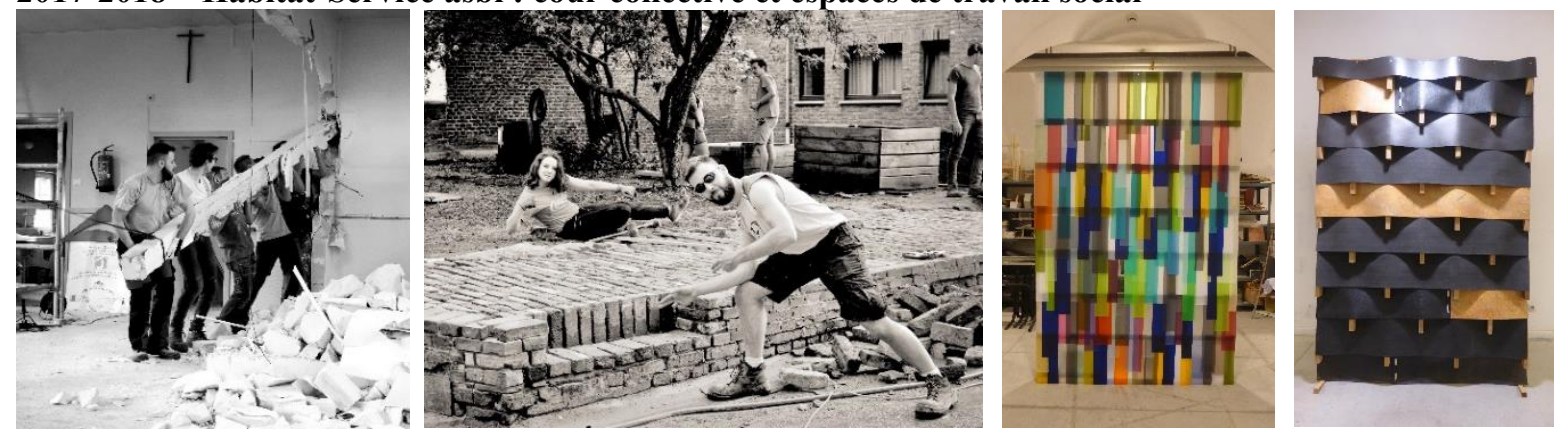

2018-2019 - Revers asbl : espaces d'ateliers intérieurs et extérieurs

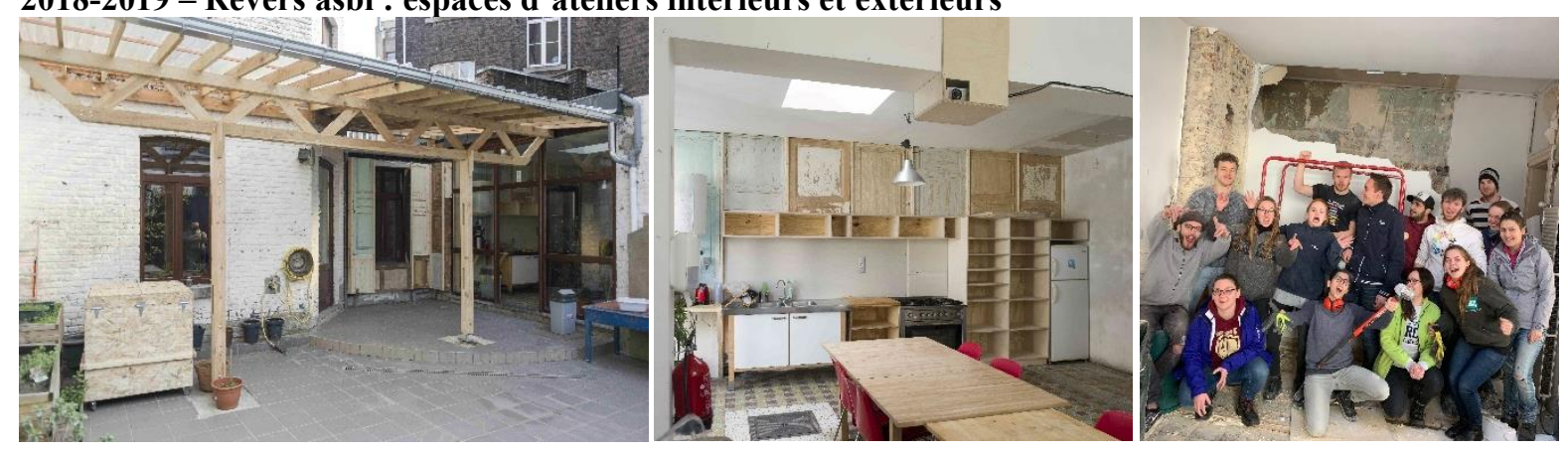

